\title{
Impact of Parallel Topical Treatment with Nadifloxacin and Adapalene on Acne Vulgaris Severity and Quality of Life: A Prospective, Uncontrolled, Multicentric, Noninterventional Study
}

\author{
Claudia Neumeister $^{\mathrm{a}}$ Rolf-Hasso Bödeker ${ }^{\mathrm{b}} \quad$ Ulrich Schwantes $^{\mathrm{a}} \quad$ Claudia Borelli $^{\mathrm{c}}$ \\ aDepartment of Medical Science/Research \& Development, Clinical Research, Dr. Pfleger Arzneimittel GmbH, Bamberg,

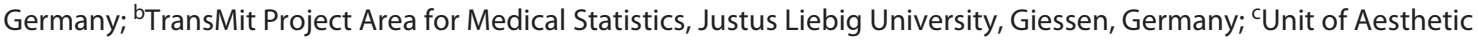 \\ Dermatology and Laser, Department of Dermatology, Eberhard Karls University, Tübingen, Germany
}

\section{Keywords}

Adapalene - Nadifloxacin · Severity of acne - Quality of life ·

Tolerability

\begin{abstract}
Introduction: Daily parallel application of adapalene and nadifloxacin has been determined to be effective and well tolerated in patients with acne vulgaris in randomized, controlled clinical studies. Here, the authors report the results from a large, prospective, uncontrolled, multicentric, noninterventional study under real-life conditions in Germany. The effect of treatment on acne severity, safety, and, for the first time, health-related quality of life (HRQoL) was investigated. Methods: Of the 292 patients (safety collective: 231 adults, 61 adolescents) who had at least grade 4 acne vulgaris on the face as per the Leeds Revised Acne Grading (LRAG), 273 (efficacy collective: 213 adults, 60 adolescents) were treated with adapalene $0.1 \%$ cream or gel and nadifloxacin $1 \%$ cream for the defined minimum of 28 days. Patients were evaluated for acne severity, acne-related facial symptoms, HRQoL, overall assessment of therapy, and safety. Results: After the median treatment duration of 37 and 38 days (adults and adolescents, respectively), 93.4\% and
\end{abstract}

$85.0 \%$ of adults and adolescents, respectively, exhibited a sustained decrease in acne severity. The LRAG decreased by at least 3 scores in $29.1 \%$ and $24.6 \%$ of female and male adults, respectively. HRQoL improved in $67.9 \%$ and $63.5 \%$ of adults and adolescents, respectively (median improvement in the Dermatology Life Quality Index scores per patient of 3.0 [female adults], 1.0 [male adults], and 2.0 for all adolescents in the Children's Dermatology Life Quality Index). Female adults were more impaired in terms of HRQoL compared to male adults. The 2 best overall efficacy ratings were provided by physicians in $79.3 \%$ and $69.5 \%$ and by patients in $68.5 \%$ and $58.3 \%$ of adult and adolescent cases, respectively. The treatment was well tolerated, as reflected in the low number of 9 mild adverse events (AEs), all of which resolved without treatment. However, 4 patients terminated the study prematurely due to AEs. Conclusion: In this study, the parallel use of adapalene and nadifloxacin for at least 5 weeks resulted in a rapid improvement in acne severity, an increase in HRQoL, and a good safety profile. Therefore, it represents a promising treatment option that offers the possibility of flexible therapy adjustment.

(c) 2021 The Author(s).

Published by S. Karger AG, Basel karger@karger.com www.karger.com/bmh

Karger $\stackrel{\text { ' }}{5}$

GOPEN ACCESS
(C) 2021 The Author(s)

Published by S. Karger AG, Basel

This is an Open Access article licensed under the Creative Commons Attribution-NonCommercial-4.0 International License (CC BY-NC) (http://www.karger.com/Services/OpenAccessLicense), applicable to the online version of the article only. Usage and distribution for commercial purposes requires written permission.
Correspondence to:

Claudia Neumeister, claudia.neumeister@dr-pfleger.de 


\section{Introduction}

Acne vulgaris is a chronic inflammatory disease that occurs most prominently at skin sites with a high density of sebaceous follicles such as the face, back, and chest [1]. Acne is often caused by an androgen-induced increased sebum production, altered keratinization, inflammation, and bacterial colonization of hair follicles by Cutibacterium (formerly Propionibacterium) acnes (C. acnes). Recent observations suggest an imbalance of the microbiota in acne [2]. Clinical features of acne include excess seborrhea, inflammatory lesions (papules and pustules), noninflammatory lesions (open and closed comedones), and various degrees of scarring [1]. Acne typically affects the health-related quality of life (HRQoL) of patients, whereby more severe symptoms of acne are associated with a greater impact on HRQoL $[3,4]$. Therefore, HRQoL is a key parameter to be considered when reviewing treatment options for individual patients $[4,5]$. Epidemiological data suggest that $>80-90 \%$ of adolescents experience acne vulgaris, with approximately $30 \%$ requiring medical intervention $[6,7]$. Goulden et al. [8] stated that acne persists beyond the age of 25 in $54 \%$ and $40 \%$ of women and men, respectively.

Treatment should aim to reduce the severity of acne as well as minimize the recurrences of skin lesions and improve appearance [3-5]. Topical agents, such as retinoids and retinoids in combination with antibiotics, are identified as the first-line therapy for mild to moderate acne treatment [5, 8-10]. Retinoids act through multiple mechanisms. They reduce cellular proliferation and differentiation of follicular keratinocytes, prevent excessive cornification and follicular blockage, and reduce the release of pro-inflammatory [11] cytokines [12]. Adapalene (CAS: 106685-40-9) is a third-generation topical retinoid derived from naphthoic acid with anti-inflammatory and comedolytic properties and a negligible systemic absorption [11]. It is known to have the best tolerability profile for this class of substances [13]. Adapalene is then provided as a gel formulation for patients with oily skin, whereas the cream formulation is recommended for those with sensitive skin [14]. Various clinical trials have shown that the combination therapy of retinoids with topical antibiotics is more effective than monotherapy [1].

Nadifloxacin (CAS: 124858-35-1) is a second-generation antibacterial fluoroquinolone, which is highly potent against aerobic and anaerobic bacteria such as Cutibacterium, Streptococcus, and Staphylococcus [15-17]. It is approved for the topical use in grades I-II acne vulgaris in parts of the European Union (Spain, Germany, Italy, and
Greece), parts of Asia (Japan and South Korea), as well as South and Central America [15-17]. Topical use results in low systemic penetration [18]. Nadifloxacin has shown a good safety and efficacy profile in several studies $[15,17,19$, 20]. A randomized, double-blind study performed in healthy volunteers found no substantial irritation potential of nadifloxacin when combined with other topical anti-acne agents (e.g., adapalene, benzoyl peroxide, azelaic acid, and isotretinoin) [21]. Two randomized, controlled studies conducted in Japan have examined the efficacy and safety of the combination therapy of adapalene $0.1 \%$ gel and nadifloxacin $1 \%$ cream compared with adapalene $0.1 \%$ gel alone. The superiority of the combination therapy was found in the reduction of inflammatory lesions, and in overall satisfaction with the drug application as judged by the physicians and patients $[20,22]$. Further, the combination therapy was very well tolerated. Less skin dryness was observed, presumably due to an alleviation of adapalene side effects by nadifloxacin. One open-label, phase III nonrandomized, noncomparative study in India demonstrated the efficacy and safety of a fixed combination [23].

Here we report the first clinical routine data that evaluated the efficacy, safety, and, additionally, the HRQoL of the parallel topical use of the 2 well-established compounds, adapalene $0.1 \%$ cream or gel and nadifloxacin $1 \%$ cream, in a large population of patients with mild to moderate inflammatory acne vulgaris in clinical practices in Germany. We hypothesize that the targeted use of nadifloxacin in addition to the standard adapalene therapy may allow for an individual therapeutic approach that provides flexible, tailored therapy for times of higher medical need and further reduces the risk of antibiotic resistance development.

\section{Materials and Methods}

\section{Study Design}

In this multicentric, prospective, noninterventional study the parallel use of the retinoid adapalene $0.1 \%$ (Dipalen ${ }^{\circledR} 1 \mathrm{mg} / \mathrm{g}$ cream or gel; Dr. Pfleger Arzneimittel GmbH, Bamberg, Germany) and the fluoroquinolone nadifloxacin $1.0 \%$ (Nadixa ${ }^{\circledR} 10 \mathrm{mg} / \mathrm{g}$ cream; Ferrer International S.A., Barcelona, Spain) was examined in patients suffering from mild to moderate inflammatory acne vulgaris in routine medical care. Due to the noninterventional nature of this study, the treatment of patients has exclusively followed the clinical routine and the judgment of the physician. The decision to prescribe both adapalene and nadifloxacin was made prior to and regardless of the decision to enroll the patient in the study. No hypothesis was tested and a descriptive statistical analysis was then performed. Data were collected at 43 dermatological practices in Germany between May 2018 and November 2018. Recruitment per site was limited to a maximum ( $\max$ ) of 10 patients to avoid bias. 


\section{Study Participants}

Eligible patients must have had a confirmed diagnosis of mild to moderate inflammatory acne vulgaris in the facial area corresponding to grade 4 or higher as per the Leeds Revised Acne Grading (LRAG) system. Patients were excluded if hypersensitivity to the active ingredients or other components of the tested drug formulations were known. Patients had no prior systemic treatment and no topical combination therapy with adapalene and nadifloxacin within the last 4 weeks. If patients regularly used nonpharmacological dermocosmetic treatments, they were advised to continue the use to exclude an influence on the study outcome.

At the time of study inclusion, patients stopped any prior topical treatment. Independent of study participation, patients presented symptoms that warranted the prescription of adapalene $0.1 \%$ (Dipalen ${ }^{\circledR} 1 \mathrm{mg} / \mathrm{g}$ cream or gel) and nadifloxacin $1 \%(\mathrm{Na}-$ dixa ${ }^{\circledR} 10 \mathrm{mg} / \mathrm{g}$ cream). The frequency and mode of application were determined by the physicians and followed the physician's routine practice. Moreover, the treatment duration was determined by the physicians, although it was requested to be at least 28 days to determine the effects of the therapy. The actual treatment duration was calculated as the time between the baseline and the final visit. Due to the noninterventional nature of the study, no drug accountability was performed. At the last visit, patients were asked whether they stuck to or changed the prescribed treatment regimen. According to the summary of product characteristics (SmPC) of Nadixa ${ }^{\circledR}$, the minimum (min) age for inclusion was set at 14 years old to allow the inclusion of adolescents who have a high medical need for treatment of acne vulgaris.

\section{Efficacy and Safety Assessments}

Data collection was performed at 2 timepoints, that is, baseline and final examination. Only patients, who were treated for at least 28 days were included in the efficacy analysis. At baseline, demographic data (age and sex) and medical history (the time of first acne appearance, acne localization, and topical pretreatment) were collected, and treatment with adapalene $0.1 \%$ and nadifloxacin $1 \%$ was prescribed.

Efficacy assessments recorded at baseline and final visit included the severity grading of facial acne using the revised LRAG system (grades 1-12), a pictorial grading scale using reference photographs [24]. As proposed by Gollnick and Orfanos on the basis of a consensus conference on acne classification in 1993 [25, 26], the following are the assessed typical symptoms of acne: comedones $\leq 20$ or $>20$; papules/pustules $<10$ or $10-20$ or $>20$; nodules "none" or $\leq 10$ or $>10$; and inflammatory lesions "none," "some," or "severe" $[25,26]$. The HRQoL in adults ( $\geq 17$ years old) was evaluated using the validated German version of the Dermatology Life Quality Index (DLQI) [27]. Since DLQI is inappropriate for children and adolescents, a special version thereof, the Children's Dermatology Life Quality Index (CDLQI), was used for adolescents aged $14-16$ years [28]. The DLQI is the most frequently used skin disease-specific patient-reported outcome instrument in terms of assessing the impairment or improvement of HRQoL in an individual patient $[4,5]$. Due to its brevity and simplicity of use, it has become popular both in clinical research and in clinical practice.

At the final visit, any change in the prescribed treatment medication with adapalene $0.1 \%$ and nadifloxacin $1 \%$, or any additional systemic or topical treatment used during the treatment period and the reason for the therapy change were documented. The physician additionally documented if, in the future, a continuation of this treatment, or a treatment change, or no treatment, is indicated or wanted by the patient. An overall assessment of therapy regarding efficacy and tolerability was further assessed by both the physician and the patient, each using one of the following responses: "very good," "good," "moderate," "poor," and "very poor."

The safety of the treatment regimen was monitored by recording adverse events (AEs). Another part of the safety assessment was the result of the overall assessment of therapy regarding tolerability. In case of premature discontinuation, the date of termination and the reason (AE, lack of acceptance, lack of efficacy, and others [free text field]) were documented.

\section{Data Management}

An electronic case report form web-based data entry system was used (Inpads GmbH, Bad Dürkheim, Germany). Each investigator received a personalized online account and created patient records upon recruitment. Data were collected in pseudonymized form, and data security was assured to be in compliance with data protection laws. The entered data were then checked for plausibility and completeness. Pursuant to the Food and Drug Administration Code of Federal Regulations (FDA CFR) 21 Part 11 standard, an audit trail was implemented. The initial data were collected on May 15, 2018; the final data entry took place on November 16, 2018. After query processing, the database was closed on February $13,2019$.

\section{Sample Size}

In the literature, no estimates were observed on the probability of an improvement in symptoms by combining therapy with adapalene and therapy with nadifloxacin. In the worst case, the unknown probability of an improvement under therapy can be 0.5 . In this worst case, if the 0.95 -confidence interval (CI) is to have a width of 0.1 around the estimator obtained from the sample, a sample size of 384 is thus required. Assuming a dropout rate of $20 \%$, a sample size of 480 patients was planned. Due to insufficient recruitment, data collection was extended via Amendment No. 1 from September 2018 to November 16, 2018. Finally, 299 patients at 44 study sites were enrolled in this study. Three patients had incomplete data for baseline and no data for the final visit. For 4 other patients, no data for the final visit were provided. Owing to insufficient data, these 7 patients were only included in the sensitivity analysis. Therefore, 292 patients who were recruited in 43 study sites could be completely analyzed with regard to efficacy and safety (shown in Fig. 1). The effect and safety of therapy could be different in adolescent and adult patients; therefore, the data from adolescent and adult patients were separately analyzed. To describe the observations made in both patient cohorts, 2 collectives, that is, the safety collective and the efficacy collective were analyzed in each cohort.

\section{Statistical Analysis}

Data were submitted to an independent statistician for analysis. The SAS 9.4 software was used for the processing of the collected data and statistical analysis. The statistical analysis of data, including the interpretation of the calculated CIs or $p$ values, was performed using descriptive methods. Due to the exploratory nature of the analysis, an alpha adjustment for multiple comparisons was not performed.

In the case of qualitative variables of interest, the distribution of the observed responses was described by computing the corre- 


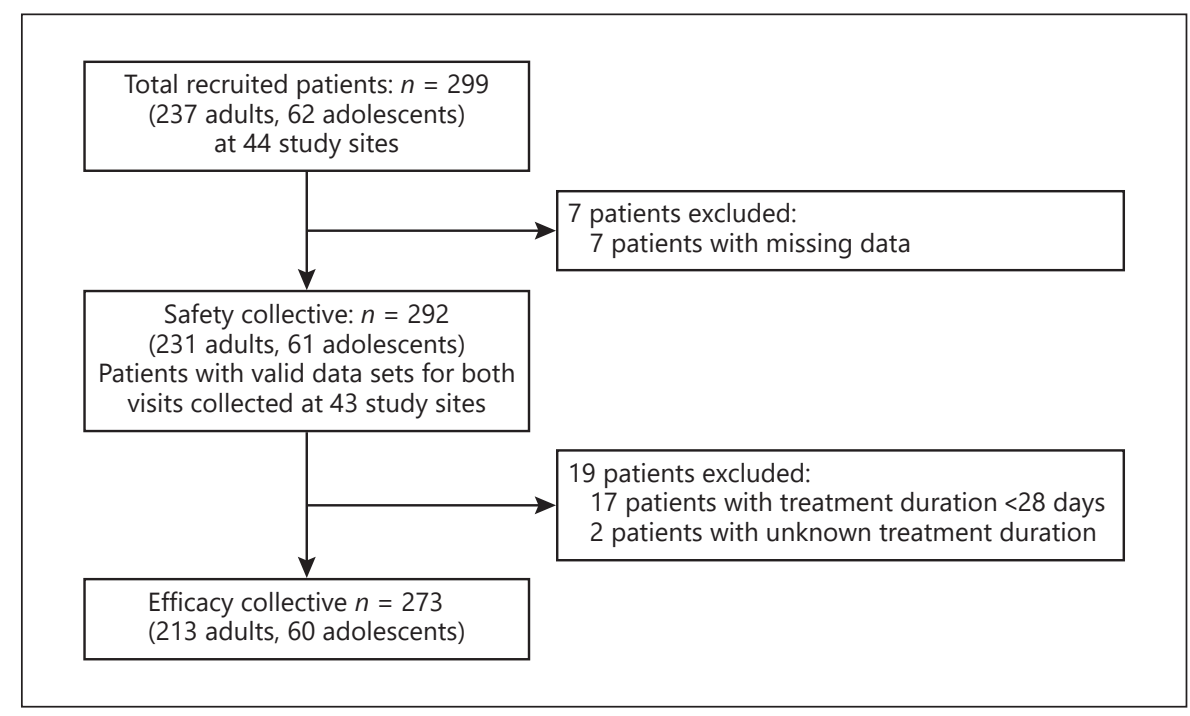

Fig. 1. Patients' flowchart.

sponding absolute and relative frequency distributions; in the case of variables on an ordinal scale, the distribution of the observed responses was described by computing the cumulative relative frequency distribution. In the case of continuous variables, the distribution of the observed responses was described in terms of extreme, quartile and median values. If normal distribution could be assumed, the mean and standard deviations were calculated. In addition, the $0.95 \mathrm{CI}$ for the estimated values was calculated. Qualitative variables were used to describe the efficacy of treatment. Logistic regression methods were then used to take the effects of possible influencing factors into account during the analysis. The collectives of adult and adolescent patients were separately analyzed. Sensitivity analyses were performed on safety variables and on efficacy variables, whereby missing variables were always replaced with the worst case. For the sensitivity analysis on efficacy, in addition to the efficacy collective, those patients were included for whom the duration of therapy was $<28$ days, and data on the assessment of the severity of acne were available at the final examination. Furthermore, patients were included for whom data on the assessment of the severity of acne were available at baseline, although not at the final examination. For the sensitivity analyses on safety, even those patients for whom no data were available regarding the assessment of tolerability by physician or patient, the occurrence of AEs or the premature discontinuation of treatment was included. For those patients, it was assumed that the assessment of tolerability was assessed to be "very poor," at least one AE occurred or that the treatment was prematurely discontinued.

\section{Results}

\section{Study Population}

In total, 299 patients at 44 study sites were included in this prospective, noninterventional study. As illustrated in Figure 1, 7 patients were excluded from the data set due to missing data, which made the data analysis infeasible, resulting in 292 patients in the safety collective. Nineteen patients were excluded from efficacy assessments since the 28-day min treatment duration was not reached (17 patients) or undeterminable ( 2 patients), thereby resulting in 273 patients in the efficacy collective. Due to the varying HRQoL questionnaires, which were inappropriate to combine due to diverging age-specific questions, the patient population was divided into adult patients aged $\geq 17$ years, and adolescents aged $14-16$ years. The efficacy collective was comprised of 213 adults and 60 adolescents; the safety collective was comprised of 231 adults and 61 adolescents.

\section{Results of the Adult Population}

Baseline Characteristics: Adult Efficacy Collective

The adult efficacy collective included 213 patients (females, 148; males, 65). Baseline characteristics for adult patients are summarized in Table 1 . The median age of this collective was 22 years (range, $17-54$ years). The median duration of acne symptoms was 15 months (females, 12 months; males, 24 months). At baseline, $93.9 \%$ of patients were confirmed to have a diagnosis of acne vulgaris severity grades $4-7$, corresponding to mild to moderate acne, and $6.1 \%$ patients had grades $8-10$, corresponding to severe acne $[5,24]$. No patient suffered from acne severity grades 11 or 12 at baseline (Table 2). Approximately half of the patients presented with $>20$ comedones, 10 20 papules or pustules, and 10 or less nodules in the facial area. Most of the patients (86.8\%) suffered from inflammatory lesions, while only $13.2 \%$ had no inflammatory lesions. The distribution of severity grades and acne symptoms was comparable between sexes. In all patients, 
Table 1. Baseline demographics of the adult efficacy collective $(n=213)$

\begin{tabular}{|c|c|c|c|}
\hline Variables & Female & Male & Total \\
\hline Gender, $n(\%)$ & $148(69.5)$ & $65(30.5)$ & $213(100)$ \\
\hline Age, median (range) & 23 years $(17-54)$ & 21 years (17-46) & 22 years $(17-54)$ \\
\hline \multirow[t]{2}{*}{ Duration of symptoms, median (range) } & 12 months (1-99) & 24 months (1-94) & 15 months (1-99) \\
\hline & Female $^{1}(n=148)$ & Male $^{2}(n=65)$ & Total $(n=213)$ \\
\hline \multicolumn{4}{|l|}{ Localization of acne, $n(\%)$} \\
\hline Face & $148(100)$ & $65(100)$ & $213(100)$ \\
\hline Chest & $54(54.5)$ & $26(40.0)$ & $80(37.6)$ \\
\hline Back & $59(39.9)$ & $42(64.6)$ & $101(47.4)$ \\
\hline Neck & $25(16.9)$ & $12(18.5)$ & $37(17.4)$ \\
\hline \multicolumn{4}{|l|}{ Leeds Revised Acne Grading system, $n$ (\%) } \\
\hline Grade 4 & $75(50.7)$ & $31(47.7)$ & $106(49.8)$ \\
\hline Grade 5 & $34(23.0)$ & $16(24.6)$ & $50(23.5)$ \\
\hline Grade 6 & $20(13.5)$ & $8(12.3)$ & $28(13.2)$ \\
\hline Grade 7 & $9(6.1)$ & $7(10.8)$ & $16(7.5)$ \\
\hline Grade 8 & $8(5.4)$ & $1(1.5)$ & $9(4.2)$ \\
\hline Grade 9 & $1(0.7)$ & $2(3.1)$ & $3(1.4)$ \\
\hline Grade 10 & $1(0.7)$ & $0(0.0)$ & $1(0.5)$ \\
\hline \multicolumn{4}{|l|}{ Acne typical facial symptoms ${ }^{3}$} \\
\hline \multicolumn{4}{|l|}{ Comedones $(n=211), n(\%)$} \\
\hline$\leq 20$ & $77(52.7)$ & $26(40.0)$ & $103(48.8)$ \\
\hline$>20$ & $69(47.3)$ & $39(60.0)$ & $108(51.2)$ \\
\hline \multicolumn{4}{|l|}{ Papules/pustules $(n=211), n(\%)$} \\
\hline$<10$ & $32(21.9)$ & $17(26.2)$ & $49(23.2)$ \\
\hline $10-20$ & $80(54.8)$ & $35(53.8)$ & $115(54.5)$ \\
\hline$>20$ & $34(23.3)$ & $13(20.0)$ & $47(22.3)$ \\
\hline \multicolumn{4}{|l|}{ Nodules ( $n=208), n(\%)$} \\
\hline None & $39(27.1)$ & $15(23.4)$ & $54(26.0)$ \\
\hline$\leq 10$ & $82(56.9)$ & $36(56.3)$ & $118(56.7)$ \\
\hline$>10$ & $23(16.0)$ & $13(20.3)$ & $36(17.3)$ \\
\hline \multicolumn{4}{|l|}{ Inflammation $(n=212), n(\%)$} \\
\hline None & $21(14.2)$ & $7(10.9)$ & $28(13.2)$ \\
\hline Some & $112(75.7)$ & $47(73.4)$ & $159(75.0)$ \\
\hline Severe & $15(10.1)$ & $10(15.6)$ & $25(11.8)$ \\
\hline \multicolumn{4}{|l|}{ Topical pretreatment ${ }^{4} n$ (\%) } \\
\hline No & $89(60.1)$ & $32(49.2)$ & $121(56.8)$ \\
\hline Yes & $59(39.9)$ & $33(50.8)$ & $92(43.2)$ \\
\hline Adapalene & $5(3.4)$ & $3(4.6)$ & $8(3.8)$ \\
\hline Azelaic acid & $17(11.5)$ & $4(6.2)$ & $21(9.9)$ \\
\hline Benzoylperoxide & $24(16.2)$ & $22(33.9)$ & $46(21.6)$ \\
\hline Clindamycin & $12(8.1)$ & $9(13.9)$ & $21(9.9)$ \\
\hline Erythromycin & $13(8.8)$ & $3(4.6)$ & $16(7.5)$ \\
\hline Isotretinoin & $3(2.0)$ & $0(0)$ & $3(1.4)$ \\
\hline Miconazole & $0(0)$ & $1(1.5)$ & $1(0.5)$ \\
\hline Natriumbituminosulfonate & $2(1.4)$ & $1(1.5)$ & $3(1.4)$ \\
\hline Salicylic acid & $5(3.4)$ & $5(7.7)$ & $10(4.7)$ \\
\hline Tretinoin & $2(1.4)$ & $2(3.1)$ & $4(1.9)$ \\
\hline Others & $2(1.4)$ & $2(3.1)$ & $4(1.9)$ \\
\hline
\end{tabular}

${ }^{1}$ Relative frequency in relation to the female collective. ${ }^{2}$ Relative frequency in relation to the male collective. ${ }^{3}$ Data not available for all patients. ${ }^{4}$ Multiple entries possible. 


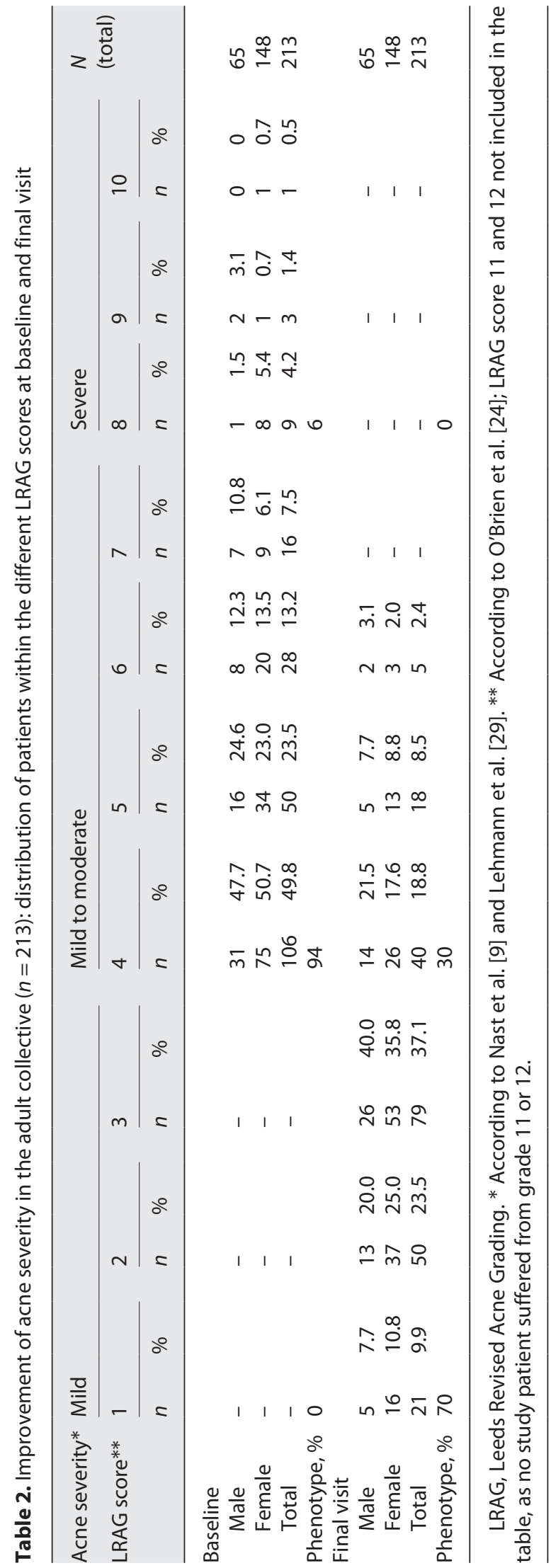

Efficacy and Tolerability of Nadifloxacin and Adapalene in Acne Vulgaris acne affected the face; however, in some patients, also the chest, the neck, and back were also affected. Males (64.6\%) had their backs more often affected with acne than females (39.9\%). In addition, $43.2 \%$ of patients had previously received $1,2,3$, or in 1 case, even 4 topical anti-acne therapeutics. Benzoyl peroxide was the most frequently used (21.6\%). Further information is shown in Table 1.

Regarding the type of adapalene application, 68.5\% $(n=146)$ and $31.5 \%(n=67)$ of patients received cream and gel, respectively. Of the 213 patients, 183 (85.9\%) applied both adapalene (cream or gel) and nadifloxacin each once per day (one product in the morning and the other in the evening). Four patients (1.9\%) applied both drugs consecutively in the evening. Twenty-six patients (12.2\%), who tended to have higher acne severity, administered one or both drugs twice a day.

The median duration of treatment was 37 days for 207 patients who completed the study as planned. Of these patients, $50 \%$ had a treatment duration between 32 and 48 days (first and third quartiles).

Efficacy Results: Adults

After a median of 37 days of treatment with adapalene $0.1 \%$ and nadifloxacin $1 \%$, a marked improvement in acne severity, acne-related symptoms, and HRQoL was observed.

Changes in the Severity of Acne Vulgaris on the Face: Adults

The vast majority of patients showed an improvement in the severity of acne symptoms on the face (199 patients, $93.4 \%)$. For 13 patients (6.1\%), the severity did not change; 1 patient showed worsening of the disease $(0.5 \%)$. An improvement of at least 3 LRAG scores was found for 59 patients (43 [29.1\%] and 16 [24.6\%] female and male patients, respectively). No major differences between the sexes were observed. Table 2 describes the detailed distribution of LRAG scores within the adult efficacy cohort at baseline and final visit. At baseline all patients suffered from mild/moderate to severe acne corresponding to LRAG scores $4-10$, with the most frequently observed LRAG scores being grade $4(n=106,49.8 \%)$ or alternative grade $5(n=50,23.5 \%)$ and grade $6(n=16,13.2 \%)$. At the final visit, a marked improvement within this collective of adult patients was observed, with the most frequently observed grades being grade $3(n=79,37.1 \%)$, grade $2(n=50,23.5 \%)$, and grade $4(n=40,18.8 \%)$. After the treatment period, $70.4 \%$ of patients $(n=150)$ were classified as having mild acne with LRAG scores 1-3, while $29.6 \%$ of patients $(n=63)$ were found to have mild 
Fig. 2. Distribution of the DLQI scores at baseline and final visit described by Boxplots*, separated by gender for those patients with data at both visits. The boxplot in the middle between baseline and final visit indicates the distribution of the observed changes during therapy - efficacy collective of adult patients. ${ }^{*}$ Box bottom line: lower quartile, top line: upper quartile, line in the box: median. Whiskers: smallest or largest score with $\leq 1.5$-fold interquartile range from the box. Small circles: extreme values. DLQI, Dermatology Life Quality Index.

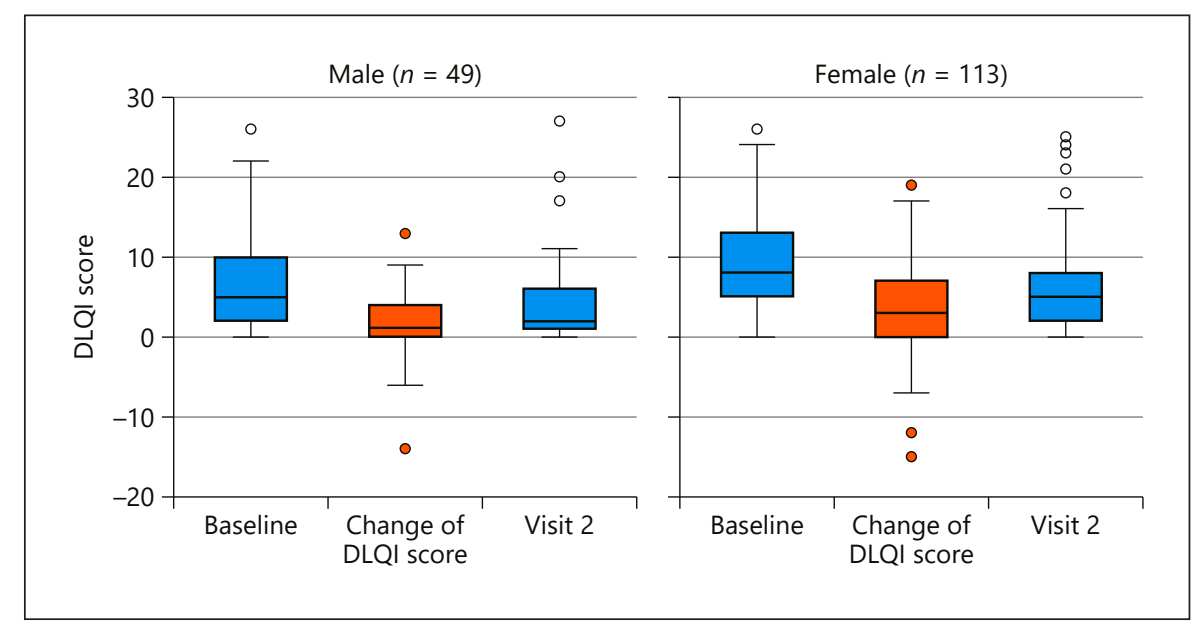

Table 3. Parameters of the distribution of the DLQI score at baseline and final visit and the change of the DLQI score observed under therapy (baseline minus final visit) separated by gender for those patients with data at both visits - efficacy collective of adult patients

\begin{tabular}{|c|c|c|c|c|c|c|}
\hline \multirow[t]{2}{*}{ Gender } & \multicolumn{6}{|c|}{$\begin{array}{l}\text { Parameters of localization and dispersion of } \\
\text { DLQI score }\end{array}$} \\
\hline & $n$ & $\min$ & $\begin{array}{l}\text { lower } \\
\text { quartile }\end{array}$ & median & $\begin{array}{l}\text { upper } \\
\text { quartile }\end{array}$ & $\max$ \\
\hline \multicolumn{7}{|l|}{ Male } \\
\hline Baseline & 49 & 0.0 & 2.0 & 5.0 & 10.0 & 26.0 \\
\hline Final visit & 49 & 0.0 & 1.0 & 2.0 & 6.0 & 27.0 \\
\hline Change & 49 & -14.0 & 0.0 & 1.0 & 4.0 & 13.0 \\
\hline \multicolumn{7}{|l|}{ Female } \\
\hline Baseline & 113 & 0.0 & 5.0 & 8.0 & 13.0 & 26.0 \\
\hline Final visit & 113 & 0.0 & 2.0 & 5.0 & 8.0 & 25.0 \\
\hline Change & 113 & -15.0 & 0.0 & 3.0 & 7.0 & 19.0 \\
\hline
\end{tabular}

DLQI, Dermatology Life Quality Index; min, minimum; max, maximum.

to moderate acne with LRAG scores 4-6 [5, 29]. A higher LRAG (grade 7 or more) was no longer represented at final visit (Table 2).

Changes in the Typical Symptoms of Acne: Adults

With the improvement of acne severity, a decrease in the number of acne symptoms was observed in most of the patients. Over the treatment period, comedones improved in 83 of 210 patients (39.5\%). Males showed a higher improvement in $47.7 \%$ of cases $(n=31 ; 0.95$ CI: [35.1\%; 60.5\%]) than females in $35.9 \%$ of cases $(n=52$, 0.95 CI: [28.1\%; 44.2\%]. The amount of patients classified with $>20$ comedones was reduced by $37.1 \%(n=78)$ after at least 4 weeks of therapy. Furthermore, $59.1 \%$ of the patients (123 of 208) presented with a decreased number of papules and pustules at the final visit. No change was observed for $38.9 \%$ of patients $(n=81)$, and only $1.9 \%$ of patients ( $n=4$, all females) showed an increased number of papules and pustules. After the treatment period, the number of patients with $>20$ papules or pustules decreased by $19.2 \%(n=40)$. For $41.0 \%$ of the patients ( 84 of 205) of the adult efficacy collective, an improvement in the symptom nodules was determined; for more than half of the patients $(57.1 \%, n=117)$, the symptom remained unchanged at the end of the treatment. In addition, $14.1 \%$ fewer patients $(n=29)$ were classified with $>10$ nodules. At the final visit, none of the patients suffered from severe inflammation anymore. From the baseline (26 patients) to the final visit (111 patients), the number of patients with no signs of inflammation increased by 85 patients. Meanwhile, 4 of the 209 patients (1.9\%) ( 3 females and 1 male) reported worsening.

\section{HRQoL: Adults}

HRQoL was analyzed in 162 patients (females, 113; males, 49), for whom data were available at baseline and at the end of the observation period. Overall, improvement in HRQoL was observed in 110 patients (67.9\%), no change in 27 patients (16.7\%), and worsening in 25 patients $(15.4 \%)$. Separately analyzing the genders, the baseline severity of acne was deemed comparable; however, the baseline median DLQI sum score was $8.0(\mathrm{~min}=0$; $\mathrm{Q} 1=5.0 ; \mathrm{Q} 3=13.0 ; \max =26.0 ; n=113$ ) for females and $5.0(\min =0 ; \mathrm{Q} 1=2.0 ; \mathrm{Q} 3=10.0 ; \max =26.0 ; n=49)$ for males (as shown in Table 3; Fig. 2). After $>5$ weeks of treatment, the median DLQI score was $5.0(\mathrm{~min}=0$; $\mathrm{Q} 1=2.0 ; \mathrm{Q} 3=8.0 ; \max =25.0 ; n=113$ ) for females and 
Table 4. DLQI results of the adult collective grouped in 5 classes, as introduced by Hazarika and Archana, based on their impact on patient life, at baseline and final visit

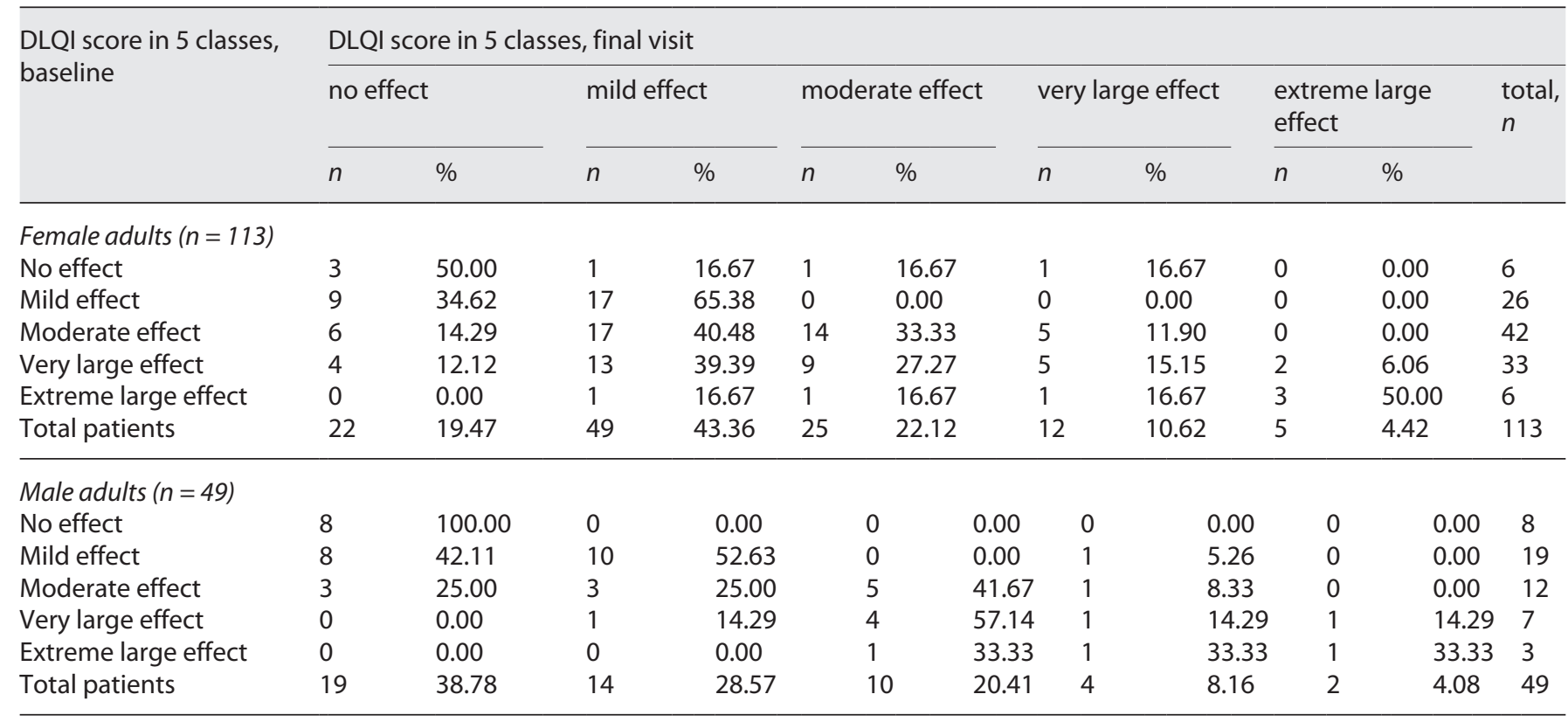

DLQI, Dermatology Life Quality Index.

$2.0(\min =0 ; \mathrm{Q} 1=1.0 ; \mathrm{Q} 3=6.0 ; \max =27.0 ; n=49)$ for males, which shows that the HRQoL of females is considerably more affected by the disease than that of males. The distribution of changes observed for each patient (DLQI score at the baseline minus DLQI score at the final visit), separated by gender, was analyzed as shown in Figure 2 . The median of the change of DLQI sum score was 3.0 $(\min =-15.0 ; \mathrm{Q} 1=0 ; \mathrm{Q} 3=7.0 ; \max =19.0 ; n=113)$ for females and $1.0(\min =-14.0 ; \mathrm{Q} 1=0 ; \mathrm{Q} 3=4.0 ; \max =$ $13.0 ; n=49$ ) for males. To make the clinical relevance of the HRQoL improvement clearer, the DLQI data were classified according to Hazarika and Archana with regard to the impact of the disease on the quality of life of patients (sum score 0-1: no effect, 2-5: mild effect, 6-10: moderate effect, 11-20: very large effect, and 21-30: extreme large effect on patient's life) (Table 4) [30]. The proportion of females who reported a great $(n=33$, $29.2 \%)$ or extreme impact $(n=6,5.3 \%)$ of acne on their lives dropped from $34.5 \%(n=39)$ to $15.0 \%(n=17)$ at the final visit. At the same time, the proportion of females who were classified with no or only a mild effect of the disease on their lives increased from $28.3 \%(n=32)$ at baseline to $62.8 \%(n=71)$ after at least 4 weeks of treatment. The HRQoL of male adults is less affected by their disease. In this study, $20.4 \%(n=10)$ of males were clas-

Efficacy and Tolerability of Nadifloxacin and Adapalene in Acne Vulgaris sified with a great $(n=7,14.3 \%)$ or extreme impact $(n=$ $3,6.1 \%)$ on their lives at baseline, a proportion that decreased to $12.2 \%(n=6)$ at the final visit. Simultaneously, $55.1 \%(n=27)$ of the males classified with no $(n=8$, $16.3 \%)$ or only a mild effect $(n=19,38.8 \%)$ on their lives at the start of the study increased to $67.4 \%(n=33)$ over the observation period. Rank correlation testing showed a clear indication of a correlation between the change of severity of acne vulgaris and the change of HRQoL in the female adult population $(r=0.33$; calculated $P<0.001$, $n=113)$.

Overall Assessment of Therapy: Adults

Using a 5-point Likert scale, the physicians and patients assessed the overall response to treatment as "good" or "very good" in $79.3 \%(n=169)$ and $68.5 \%(n=146)$ of cases and as "poor" or "very poor" in $2.8 \%(n=6)$ and $8.0 \%(n=17)$ of cases, respectively (data not shown). Tolerability was assessed as "good" or "very good" in $84.7 \%$ ( $n=177)$ (physicians) and $75.1 \%(n=157)$ (patients) of cases, and as "poor" or "very poor" in $1.4 \%(n=3)$ and $4.3 \%(n=9)$ of cases, respectively.

The continuation of the parallel treatment regimen or treatment with at least one of the 2 drugs, was planned in $69.5 \%(n=148)$ and $11.7 \%(n=25)$ of cases, respectively. 
A switch in treatment was then performed in $7.5 \%$ of patients $(n=16)$, and no further treatment was required in $11.3 \%$ of patients $(n=24)$.

\section{Additional Treatment: Adults}

In total, 28 patients $(13.1 \%)$ of the efficacy group of adults (males, 10; females, 18) received an additional treatment (4 [1.9\%], 3 [1.4\%], and 22 patients [10.3\%] underwent systemic, topical, and cosmetic treatments, respectively).

AEs, Premature Termination: Adult Safety Collective

$\mathrm{AE}$ data were available for 229 of the 231 adults. In total, 8 mild AEs were reported in 4 patients $(1.8 \%$, CI: $[0.5 \% ; 4.4 \%])$. All were facial skin irritations, judged as probably or possibly related to the study medication, except for one ("burning eyes," unlikely related to the study medication). Serious AEs were not reported. All events spontaneously resolved. The relative frequencies of the occurrence of AEs were in or below the range of those listed in the SmPCs of both compounds. Three patients prematurely terminated from the study due to AEs. Another 6 patients discontinued the study due to the lack of efficacy and 1 patient due to the loss of medication.

\section{Results of the Adolescent Population}

Baseline Characteristics: Adolescent Efficacy

Collective

The smaller subgroup of 60 adolescents (males, 33; females, 27; aged 14-16 years) suffered from acne symptoms for a duration of 1-48 months, whereby most of the patients were diagnosed between 6 and 12 months (39.4\%) and between 12 months and 24 months (27.3\%) before baseline. The frequency of location, the frequency of the typical symptoms of acne, and the distribution of the LRAG score were comparable between the collective of the adolescent and the adult patients. The highest severity grade observed within the adolescent collective in 2 patients was grade 9 at baseline. Compared with the adult population, only $23.3 \%(n=14)$ of the adolescent population had received topical pretreatment. Treatment changes were mostly due to the lack of efficacy. As seen in adult patients, no indication of a different distribution of the severity of the symptoms of acne between male and female patients could be found. At baseline the largest proportion of the adolescent patients suffered from $>20 \mathrm{com}-$ edones $(65.0 \%, n=39), 10-20$ papules/pustules $(48.3 \%$, $n=29), \leq 10$ nodules $(50.0 \%, n=30)$, and some inflammation $(75.0 \%, n=45)$. Forty-nine patients received both adapalene $0.1 \%$ and nadifloxacin $1 \%$ once daily, whereas
Table 5. Parameters of the distribution of the CDLQI score at baseline and final visit and the observed change of the CDLQI score under therapy (baseline minus final visit) separated by gender for those patients with data at both visits - efficacy collective of adolescent patients

\begin{tabular}{|c|c|c|c|c|c|c|}
\hline \multirow[t]{2}{*}{ Gender } & \multicolumn{6}{|c|}{$\begin{array}{l}\text { Parameters of localization and dispersion of CDLQI } \\
\text { score }\end{array}$} \\
\hline & $n$ & $\min$ & $\begin{array}{l}\text { lower } \\
\text { quartile }\end{array}$ & median & $\begin{array}{l}\text { upper } \\
\text { quartile }\end{array}$ & $\max$ \\
\hline \multicolumn{7}{|l|}{ Male } \\
\hline Baseline & 29 & 0.0 & 1.0 & 7.0 & 9.0 & 24.0 \\
\hline Final visit & 29 & 0.0 & 1.0 & 3.0 & 6.0 & 18.0 \\
\hline Change & 29 & -3.0 & 0.0 & 2.0 & 4.0 & 8.0 \\
\hline \multicolumn{7}{|l|}{ Female } \\
\hline Baseline & 23 & 0.0 & 4.0 & 8.0 & 15.0 & 22.0 \\
\hline Final visit & 23 & 0.0 & 2.0 & 4.0 & 9.0 & 22.0 \\
\hline Change & 23 & -1.0 & 0.0 & 2.0 & 7.0 & 17.0 \\
\hline
\end{tabular}

CDLQI, Children's Dermatology Life Quality Index; min, minimum; max, maximum.

11 patients received $>2$ applications per day. In total, 43 patients $(71.2 \%)$ received Dipalen ${ }^{\circledR}$ cream, and 17 patients $(28.8 \%)$ received Dipalen ${ }^{\circledR}$ gel.

\section{Efficacy Results: Adolescents}

The severity of acne (LRAG scores) improved with therapy in 51 adolescents (85.0\%, 95\% CI: [73.4; 92.9]) and remained unchanged in 9 adolescent patients (15.0\%). A worsening of the disease did not occur. The improvement of the typical symptoms of acne with therapy ranged between $45.0 \%$ for the symptom papules/pustules and $33.3 \%$ for the symptom comedones. Inflammation decreased in $43.3 \%$ of adolescent patients.

For the HRQoL assessment, the CDLQI, a modified version of the DLQI questionnaire for children and adolescents up to 16 years was used, as recommended by Marron et al. [31]. The CDLQI covers 6 areas of daily activities, including symptoms and feelings, leisure, school or holidays, personal relationships, sleep, and treatment. Although the evaluation is similar to the adult version (DLQI) with a max score of 30, it is inappropriate to combine the results of both questionnaires as they are structured differently and ask different questions [31]. Overall, the HRQoL improved in 63.5\% $(n=33)$ of the adolescent patients, remained unchanged in $21.1 \%(n=11)$, and worsened in $15.4 \%(n=8)$.

As determined for 52 adolescents, for whom the assessment was conducted at both visits, the median CDLQI 
Fig. 3. Distribution of the CDLQI scores at baseline and final visit described by Boxplots, separated by gender for those patients with data at both visits. The boxplot in the middle between baseline and final visit indicates the distribution of the observed changes during therapy - efficacy collective of adolescent patients. CDLQI, Children's Dermatology Life Quality Index.
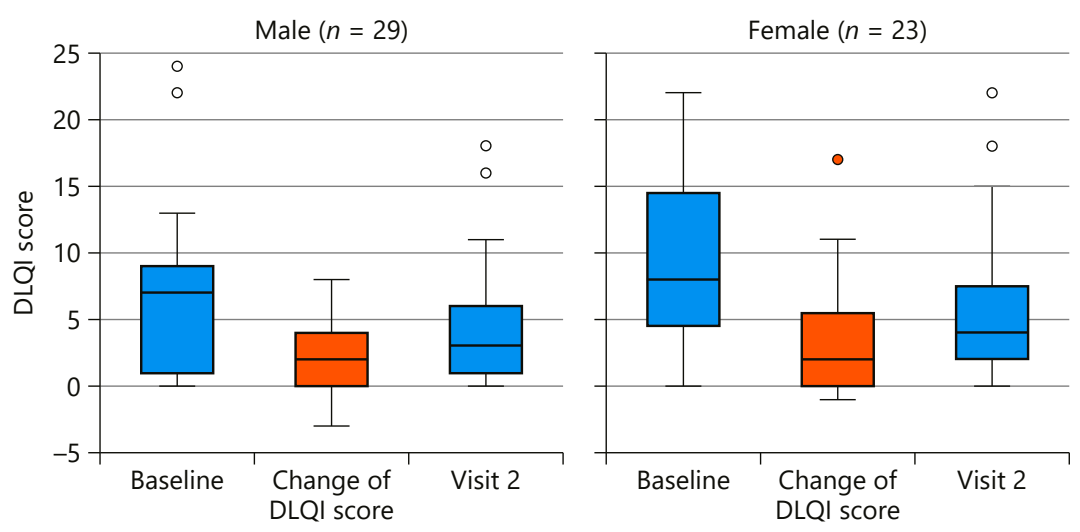

score at baseline was $7.0(\min =0 ; \mathrm{Q} 1=1.0 ; \mathrm{Q} 3=9.0$; $\max =24.0 ; n=29)$ for males and $8.0(\min =0 ; \mathrm{Q} 1=4.0$; $\mathrm{Q} 3=15.0 ; \max =22.0 ; n=23$ ) for females. At the final visit, the median CDLQI score decreased by 4 scores in both genders, to $3.0(\min =0 ; \mathrm{Q} 1=1.0 ; \mathrm{Q} 3=6.0 ; \max =18.0 ; n$ $=29)$ for males and $4.0(\min =0 ; \mathrm{Q} 1=2.0 ; \mathrm{Q} 3=9.0 ; \mathrm{max}=$ $22.0 ; n=23$ ) for females. The median distribution of observed changes in the CDLQI score during therapy (from the baseline visit to the final visit) was 2.0 for both the male and female adolescent patients (as shown in Table 5; Fig. 3).

If classified according to Hazarika and Archana [30], as described above for the adult collective, only 2 and 4 males $(n=6,20.7 \%)$, and 1 and 8 females $(n=9,39.1 \%)$ at baseline were classified as having an extreme or very great effect of the disease on the HRQoL, respectively (data not shown). At the final visit, no male presented with an extreme effect; but 1 female (4.35\%). Further, 3 males $(10.3 \%)$ and 4 females (17.4\%) experienced a very large effect of acne on their HRQoL. The proportion of male adolescents with no or only a mild effect on HRQoL increased from $48.2 \%(n=14)$ to $69.0 \%(n=20)$ and from $30.4 \%(n=7)$ to $69.6 \%(n=16)$ in females. Female adolescents were, therefore, more affected by HRQoL impairment than male adolescents.

\section{Overall Assessment of Therapy: Adolescents}

In 41 (69.5\%) and 35 (58.3\%) cases, physicians and patients, respectively, assessed the overall response to treatment as "good" or "very good" and in 4 (6.8\%) and 6 (10.0\%) cases as "poor" or "very poor" respectively. Tolerability was rated as "good" or "very good" in 50 (84.7\%) and $47(78.7 \%)$ cases by physicians and patients and as "poor" or "very poor" in 4 (6.8\%) and $6(10.0 \%)$ of cases, respectively.

Efficacy and Tolerability of Nadifloxacin and Adapalene in Acne Vulgaris
AEs, Premature Termination: Adolescent Safety

Collective

One of the 61 adolescent patients (1.6\%, CI: [0.04\%; $8.80 \%]$ ) suffered from "swollen eyelid, burning sensation, and facial swelling" in relation with the application of adapalene and, therefore, prematurely terminated the study. Another patient prematurely terminated the study after 46 days due to the lack of acceptance.

\section{Discussion}

For the purpose of providing further scientific evidence on the parallel use of nadifloxacin and adapalene in clinical routine, the authors conducted this noninterventional study demonstrating efficacy and safety in the treatment of mild to moderate inflammatory acne vulgaris in adults and adolescents in Germany. To the best of our knowledge, the authors are the first to investigate the effect of that treatment on HRQoL by using age-specific, self-administered, validated questionnaires.

To assess the disease activity, the authors used severity grading using the LRAG score in combination with lesion counting, which are both considered to be precise, objective, and highly discriminative assessment tools [32]. The daily parallel treatment for approximately 5 weeks (median for adults: 5.3 weeks; median for adolescents: 5.4 weeks) has led to an improvement in acne severity in the vast majority of patients and of note, to a decrease of at least 3 LRAG scores in more than a quarter of the enrolled patients (both adults and adolescents). Although patients had at least LRAG scores of 4 at baseline, nearly 3 quarters of adults and adolescents presented with LRAG scores 1-3 at the final visit, which corresponds to mild acne [5]. Al- 
though this classification by acne phenotypes may only be a limited approach, as stated by the authors, the LRAG classification was noted to correlate well with the assessment of the typical symptoms of acne in our study. At the final visit, none of the patients had severe inflammation anymore; the number of patients with no inflammation increased by $39.7 \%$. Concurrently, the number of papules and pustules decreased in approximately $60 \%$ and $45 \%$ of adults and adolescents, respectively; in addition, the number of comedones decreased in $40 \%$ and $33 \%$ of adults and adolescents, respectively. Although the results are not directly comparable due to use of varying assessment methods, this seems to be consistent with the results from a previous observational study with 117 patients using adapalene and nadifloxacin as a fixed combination, which reported a reduction in inflammatory and noninflammatory lesions by $46 \%$ and $50 \%$, respectively, after 4 weeks [24] of treatment [23]. Although adapalene alone is known to act as a comedolytic, to resolve the precursor microcomedone lesions, and to have anti-inflammatory properties, its use in combination with an antibacterial agent that reduces follicular colonization with $C$. acnes appears to enhance the efficacy relative to the use of the retinoid alone. By targeting these significant pathophysiological pathways, both compounds appear to have an additive and complementary effect [33]. In a randomized controlled study by Takigawa et al. [22], the combination of adapalene and nadifloxacin was superior to adapalene monotherapy in reducing inflammatory lesions after 4 weeks ( $45 \%$ compared with $24 \%$, respectively), a trend that continued up to 12 weeks. The authors of the European evidence-based (S3) guideline agreed that a new treatment option must achieve at least a $10 \%$ greater reduction in acne-related symptoms relative to a comparator treatment in order to be considered as clinically relevant [5]. Although we did not include a control arm in this study, our results support the findings of the controlled study by Takigawa et al. [22], which achieved the necessary $10 \%$ reduction in acne symptoms and demonstrated that the parallel application of adapalene and nadifloxacin is a promising, clinically relevant treatment strategy for mild to moderate inflammatory acne vulgaris.

Several acne guidelines have recommended the assessment of HRQoL in clinical studies as this is the most relevant aspect for patients in their daily life. Our study is the first to investigate the impact of the combination of adapalene and nadifloxacin on the HRQoL. The authors found an overall improvement in approximately twothirds of the study population (both adults and adolescents), and a median change in the DLQI score of 3.0 in the largest collective of female adults, 1.0 for the male adults and 2.0 for both genders in the adolescent collective over the study period. According to Marron et al. [31], a change in at least 4 points in the DLQI score in inflammatory skin conditions is considered clinically relevant. In this study, 215 patients completed the DLQI at baseline and final visit. If the 37 patients who had a score $<4$ at baseline, which represents no or only a mild effect on the HRQoL from the beginning, are not taken into account, a clinically relevant change in the score by at least 4 points can be observed for $48.0 \%$ of adult patients. The CDLQI was then completed at baseline and final visit by 52 adolescent patients. At baseline, 16 adolescents had a score $<4$. Accordingly, if these are not taken into account, a clinically relevant improvement in the HRQoL by at least 4 score points can be observed for $50.0 \%$ of the adolescent patients. Classification according to the influence of acne on patients' lives showed that, in all collectives, the proportion of patients whose symptoms had none or only a slight effect on the HRQoL increased during the study period (increase in the adult collective, $34.5 \%$ more females and $12.3 \%$ more males; adolescent collective, $39.2 \%$ more females and $20.8 \%$ more males). These are remarkable findings since the duration of treatment was significantly shorter in comparison with other acne studies, wherein patients were often treated for 12 weeks or longer (median duration of treatment/adults: 5.3 weeks, third quartile 6.9 weeks; adolescents: median 5.4 weeks, third quartile 7.1 weeks). To compare our results in terms of the HRQoL with other topically used compounds in acne therapy, the study of Kainz et al. [34] investigated the effect of azelaic acid 20\% cream on the HRQoL in adult female patients [35]. The median DLQI score decreased from 9.0 at the start of treatment, to 7.0 after 4-8 weeks, and to 5.0 after 12 weeks of treatment. Another observational study of concomitant clindamycin and tretinoin treatment in acne patients reported a mean DLQI change from baseline to week 12 of minus 3.4 scores [35]. Although both studies are not directly comparable due to different settings, a median change of minus 2.0 in the DLQI score for both adults and adolescents in our study seems to be equivalent. Although the severity of acne in female and male adults did not differ, a remarkable gender difference in the HRQoL was observed. The DLQI score reported by females was already 3 points higher at the start of the study than that of males, a trend that persisted until the final visit. This outcome confirms previous findings by Berg et al. [36] and implies that female patients seem to suffer more from their disease than male patients. 
The overall applicability of the study is limited by the nature of its noninterventional design, which includes the lack of a control group and a prespecified hypothesis, and the absence of statistical power for certain analysis (e.g., the investigation of a correlation between acne severity and HRQoL), which was not achieved due to insufficient patient recruitment (480 planned patients). A further limitation may be the short observation period (median treatment duration of adults: 5.3 weeks; adolescents: 5.4 weeks), as other studies regularly report data for 12 weeks or longer. With adapalene monotherapy, a clinically relevant symptom improvement in acne patients is to be expected at the earliest time (after 4 weeks), and improvement continues with further treatment [37].

In addition to the promising efficacy data, the parallel use of adapalene and nadifloxacin was very well tolerated. Only nonserious, mild AEs were reported in $1.8 \%$ and $1.6 \%$ of adults and adolescents, respectively, a rate lower than that listed in the SmPCs of both topicals. In addition, we observed a low rate of premature termination of treatment (adults: $2.8 \%$, adolescents: $3.3 \%$ ), which implies good patient adherence due to the good safety of the treatment regimen. Our results are consistent with that of Takigawa et al. [22], wherein patients who received adapalene and nadifloxacin experienced mostly mild symptoms of burning and dryness, and only a few suffered from severe skin irritations. When compared with adapalene monotherapy, significantly fewer patients suffered from dryness, and only one patient withdrew due to a severe skin irritation. A study by Kobayashi et al. [20] found that the addition of nadifloxacin to adapalene does not lead to an increase in side effects of erythema and scaling, although it is superior in terms of severity of the observed erythema. Based on these results, it has been postulated that nadifloxacin may alleviate the side effects of adapalene. These previous studies and our data suggest that there should be no great concern regarding the increased irritation from the daily parallel use of the 2 therapies.

Topical antibiotics have been shown to be effective in the treatment of mild to moderate acne vulgaris. However, an increasing challenge is the development of antibiotic resistances, which may result in treatment failure. Current guidelines of care for acne emphasize strategies that reduce dependence on antibiotics and minimize the risk for resistance. In double-blind, multinational, phase III studies, nadifloxacin $1 \%$ cream has shown equal efficacy and safety as clindamycin $1 \%$ gel and erythromycin $2 \%$ cream $[38,39]$. Differences were observed in the bacteriological profile, since only an extremely low number of resistant bacteria were detected in patients treated with nadifloxacin in comparison to patients treated with erythromycin [39]. This result is supported by an in vitro study that investigated the resistance of acne pathogens (C. acnes, methicillin-susceptible and -resistant Staphylococcus aureus and Staphylococcus epidermidis) to various antibiotic substances collected in several European Union countries [40]. Nadifloxacin was identified as the most potent antibiotic, and no increase in resistance to nadifloxacin was observed in the pathogens collected in Germany, wherein nadifloxacin has been in use in the previous 2 years [40]. These results could recently be confirmed by an in vitro study assessing the activity of nadifloxacin against C. acnes compared with those of erythromycin, clindamycin and tetracycline [41]. Therefore, nadifloxacin seems to be the better choice due to its efficacy and very low rate of resistance development. The European guideline for the treatment of acne does not recommend topical antibiotics for comedonal acne but for papulopustular acne. In our opinion, in future clinical practice, adapalene could be applied as an introductory standard therapy, which is supplemented with nadifloxacin at times of acute inflammatory conditions for adequate control. However, due to the challenge of resistance formation, the use of nadifloxacin should frequently be well considered and limited to the necessary time period. Parallel treatment, as described in this study, enables such controlled use of nadifloxacin.

\section{Conclusion}

The ideal acne treatment should quickly and effectively control the symptoms of acne, have few side effects, and should be well accepted by the patients. Our results suggest that the parallel application of adapalene and nadifloxacin is a promising treatment option in adults and adolescents with acne vulgaris, with a rapid onset of action and considerable improvement of acne symptoms and HRQoL after at least 5 weeks of treatment. Future long-term studies in a larger number of patients including a comparator treatment are required.

\section{Acknowledgments}

We thank Carola Effertz who participated in writing and in the technical editing of this manuscript, which is funded by Dr. Pfleger Arzneimittel $\mathrm{GmbH}$, Germany. We would also like to extend our gratitude to Anna Derr (Department Medical Science, Dr. Pfleger Arzneimittel $\mathrm{GmbH}$, Bamberg, Germany) for proofreading the manuscript. 


\section{Statement of Ethics}

The research was conducted ethically in accordance with the World Medical Association's Declaration of Helsinki. The study was conducted in compliance with $₫ 67$ (6) of the German Drug Law, in accordance with the joint recommendations for the planning, conduct, and analysis of observational studies, compiled by the Federal Institute for Drugs and Medical Devices (BfArM) and the Paul Ehrlich Institute (edition of July 7, 2010). Further, the German guidelines and recommendations for the assurance of good epidemiological practice (GEP) were applied [42]. The investigators informed the patients verbally and in writing as regards the noninterventional nature of the study and, in particular, about the handling of their data (data collection and storage, pseudonymized data dissemination, and the possibility of data inspection by monitor) before they were included in this study. Written informed consent with regard to data processing was received from all participants, and in the case of adolescents, additionally from the parents, legal guardian, or next of kin. The study obtained approval from the responsible Ethics Committee on May 5, 2018 (EC of Bavarian State Medical Association, EC No. 17087) and was registered at Deutsches Register Klinische Studien (DRKS, ID No. DRKS00014741) prior to the start. The manuscript was written in accordance with the STROBE statement (guidelines for reporting results from noninterventional studies) [43].

\section{Conflict of Interest Statement}

Claudia Neumeister is an employee and Ulrich Schwantes is a former employee of Dr. Pfleger Arzneimittel GmbH. Rolf-Hasso Bödeker performed statistical planning and analysis, funded by Dr. Pfleger Arzneimittel GmbH. Claudia Borelli received payment from Dr. Pfleger Arzneimittel GmbH for advisory services as regards the study design, results, and writing in the context of this study.

\section{Funding Sources}

This study was sponsored by Dr. Pfleger Arzneimittel GmbH, Bamberg, Germany. The funding body participated in the design and organization of the study and sponsored data collection, analysis, and writing of the manuscript.

\section{Author Contributions}

Claudia Neumeister contributed to the study methodology and design, organized the study (among other regulatory activities, choice of data entry system, recruitment and supervision of study sites, and review of final report), and reviewed the manuscript. Rolf-Hasso Bödeker contributed to the study design, performed data analysis including preparation of statistical report, and reviewed the manuscript. Claudia Borelli contributed to the study design, acted in an advisory capacity, and reviewed the manuscript. Ulrich Schwantes contributed to the study methodology and design and reviewed the manuscript. All authors have met the International Committee of Medical Journal Editors criteria for authorship for this manuscript and have taken responsibility for the integrity of the work presented and have reviewed and/or revised the manuscript. All authors have given final approval to the published version.

\section{Data Availability Statement}

All data generated or analyzed during this study are included in this article. Further enquiries can be directed to the corresponding author.

\section{References}

1 Williams HC, Dellavalle RP, Garner S. Acne vulgaris. Lancet. 2012;379(9813):361-72.

2 O'Neill AM, Gallo RL. Host-microbiome interactions and recent progress into understanding the biology of acne vulgaris. Microbiome. 2018;6(1):177.

3 Fabbrocini G, Cacciapuoti S, Monfrecola G. A qualitative investigation of the impact of acne on Health-Related Quality of Life (HRQL): development of a conceptual model. Dermatol Ther. 2018 Mar;8(1):85-99.

4 Tasoula E, Gregoriou S, Chalikias J, Lazarou D, Danopoulou I, Katsambas A, et al. The impact of acne vulgaris on quality of life and psychic health in young adolescents in Greece: results of a population survey. Anais Brasileiros de Dermatologia. 2012 Dec;87(6):862-9.

5 Nast A, Dréno B, Bettoli V, Degitz K, Erdmann R, Finlay AY, et al. European evidencebased (S3) guidelines for the treatment of acne. J Eur Acad Dermatol Venereol. 2012;26 Suppl 1:1-29.
6 Tan JKL, Bhate K. A global perspective on the epidemiology of acne. Br J Dermatol. 2015; 172:3-12. Available from: http://doi.wiley. com/10.1111/bjd.13462.

7 Ayer J, Burrows N. Acne: more than skin deep. Postgrad Med J. 2006 Aug;82(970):5006.

8 Goulden V, Stables GI, Cunliffe WJ. Prevalence of facial acne in adults. JAAD 1999; 41(4):577-580.

9 Nast A, Bayerl C, Borelli C, Degitz K, Dirschka T, Erdmann R, et al. Behandlung der Akne. Deutsche Dermatologische Gesellschaft. 2011. AWMF No. 013/017.

10 Thiboutot DM, Dréno B, Abanmi A, Alexis AF, Araviiskaia E, Barona Cabal MI, et al. Practical management of acne for clinicians: An international consensus from the global alliance to improve outcomes in acne. J Am Acad Dermatol. 2018 Feb 1;78(2):S1-23.e1.
11 Rusu A, Tanase C, Pascu GA, Todoran N. Recent advances regarding the therapeutic potential of adapalene. Pharmaceuticals. 2020 Aug 28;13(9):217.

12 Kraft J, Freiman A. Management of acne. CMAJ. 2011;183(7):E430-5.

13 Kolli SS, Pecone D, Pona A, Cline A, Feldman SR. Topical retinoids in acne vulgaris: a systematic review. Am J Clin Dermatol. 2019;20: 345-65.

14 Latter G, Grice JE, Mohammed Y, Roberts MS, Benson HAE. Targeted topical delivery of retinoids in the management of acne vulgaris: current formulations and novel delivery systems. Pharmaceutics. 2019 Sep 24;11(10):490.

15 Jacobs MR, Appelbaum PC. Nadifloxacin: a quinolone for topical treatment of skin infections and potential for systemic use of its active isomer, WCK 771. Expert Opin Pharmacother. 2006 Oct;7(14):1957-66. 
16 Alba V, Urban E, Angeles Dominguez M, Nagy E, Nord CE, Palacín C, et al. In vitro activity of nadifloxacin against several Grampositive bacteria and analysis of the possible evolution of resistance after 2 years of use in Germany. Int J Antimicrob Agents. 2009 Mar; 33(3):272-5.

17 Schöfer H, Göllner A, Kusche W, Schwantes U. Effectiveness and tolerance of topical nadifloxacin in the therapy of acne vulgaris (grade I-II): results of a non-interventional trial in 555 patients. J Appl Res. 2009;9(3):44-51.

18 Jacobs MR, Appelbaum PC. Nadifloxacin: a quinolone for topical treatment of skin infections and potential for systemic use of its active isomer, WCK 771. Expert Opin Pharmacother. 2006 Oct;7(14):1957-66.

19 Choudhury S, Chatterjee S, Sarkar DK, Dutta RN. Efficacy and safety of topical nadifloxacin and benzoyl peroxide versus clindamycin and benzoyl peroxide in acne vulgaris: a randomized controlled trial. Indian J Pharmacol. 2011;43(6):628-31.

20 Kobayashi M, Nakagawa T, Fukamachi K, Nakamura M, Tokura Y. Efficacy of combined topical treatment of acne vulgaris with adapalene and nadifloxacin: a randomized study. J Dermatol. 2011 Dec;38(12):1163-6.

21 Wilhelm KP, Wilhelm D, Neumeister C, Zsolt I, Schwantes U. Lack of irritative potential of nadifloxacin $1 \%$ when combined with other topical anti-acne agents. Clin Exp Dermatol. $2012 \mathrm{Mar} ; 37(2): 112-7$.

22 Takigawa M, Tokura Y, Shimada S, Furukawa F, Noguchi N, Ito T, et al. Clinical and bacteriological evaluation of adapalene $0.1 \%$ gel plus nadifloxacin $1 \%$ cream versus adapalene $0.1 \%$ gel in patients with acne vulgaris. J Dermatol. 2013 Aug;40(8):620-5.

23 Shah BJ, Sumathy TK, Dhurat RS, Torsekar RG, Viswanath V, Mukhi JI, et al. Efficacy and tolerability of topical fixed combination of nadifloxacin $1 \%$ and adapalene $0.1 \%$ in the treatment of mild to moderate acne vulgaris in indian patients: a multicenter, open-labelled, prospective study. Indian J Dermatol. 2014;59(4):385-9.
24 O'Brien SC, Lewis JB, Cunliffe WJ. The Leeds Revised Acne Grading system. J Dermatol Treat. 1998;9(4):215-20.

25 Pochi PE, Shalita AR, Strauss JS, Webster SB, Cunliffe WJ, Irving Katz H, et al. Report of the consensus conference on acne classification Washington, D.C., March 24 and 25, 1990. J Am Acad Dermatol. 1991;24(3):495-500.

26 Cunliffe W, Gollnick H. Akne: Klinik, Differentialdiagnose, Pathogenese, Therapie. Stuttgart: Hippokrates-Verlag; 1993.

27 Finlay AY, Khan GK. Dermatology Life Quality Index (DLQI): a simple practical measure for routine clinical use. Clin Expe Dermatol. 1994;19(3):210-6.

28 Lewis-Jones MS, Finlay AY. The Children's Dermatology Life Quality Index (CDLQI): initial validation and practical use. Br J Dermatol. 2010 Sep 6;132(6):942-9.

29 Lehmann H, Robinson K, Andrews JS, Holloway V, Goodman SN. Acne therapy: a methodologic review. J Am Acad Dermatol. 2002;47(2):231-40.

30 Hazarika N, Archana M. The psychosocial impact of acne vulgaris. Indian J Dermatol. 2016 Sep-Oct;61(5):515-20. Available from:

31 Marron SE, Chernyshov PV, Tomas-Aragones L. Quality-of-life research in acne vulgaris: current status and future directions. Am J Clin Dermatol. 2019;20:527-38.

32 Agnew T, Furber G, Leach M, Segal L. A comprehensive critique and review of published measures of acne severity. J Clin Aesthet Dermatol. 2016;9(7):40.

33 Leyden J, Stein-Gold L, Weiss J. Why topical retinoids are mainstay of therapy for acne. Dermatol Ther. 2017;7(3)293-304.

34 Kainz JT, Berghammer G, Auer-Grumbach P, Lackner V, Perl-Convalexius S, Popa R, et al. Azelaic acid $20 \%$ cream: effects on quality of life and disease severity in adult female acne patients. J Dtsch Dermatol Ges. 2016 Dec; 14(12):1249-59.

35 Ohlson J, Dakovic R, Berg M. Observational study of clindamycin phosphate and tretinoin gel for the treatment of acne. J Drugs Dermatol. 2019 Apr;18(4):328-34.
36 Berg M, Lindberg M. Possible gender differences in the quality of life and choice of therapy in acne. J Eur Acad Dermatol Venereol. 2011 Aug;25(8):969-72.

37 Jacobs A, Starke G, Rosumeck S, Nast A. Systematic review on the rapidity of the onset of action of topical treatments in the therapy of mild-to-moderate acne vulgaris. Br J Dermatol. 2014 Mar;170(3):557-64.

38 Anbarasi S, Meenakshi B, Ramya JE. Comparative study of efficacy and safety of clindamycin $1 \%$ gel and nadifloxacin $1 \%$ cream in patients with mild to moderate acne vulgaris. IOSR J Dent Med Sci. 2017;16:12-22.

39 Plewig G, Holland KT, Nenoff P. Clinical and bacteriological evaluation of nadifloxacin $1 \%$ cream in patients with acne vulgaris: a double-blind, phase III comparison study versus erythromycin 2\% cream. Eur J Dermatol. 2006;16(1):48-55.

40 Alba V, Urban E, Angeles Dominguez M, Nagy E, Nord CE, Palacín C, et al. In vitro activity of nadifloxacin against several Grampositive bacteria and analysis of the possible evolution of resistance after 2 years of use in Germany. Int J Antimicrob Agents. 2009 Mar; 33(3):272-5

41 Nenoff P, Koch D, Krüger C, Neumeister C, Götz M, Schwantes U, et al. Activity of nadifloxacin, and three other antimicrobial agents against Cutibacterium acnes isolated from patients with acne vulgaris. J Eur Acad Dermatol Venereol. 2021 Oct;35(10):e682-4.

42 Hoffmann Wolfgang, Latza Ute, Baumeister Sebastian E., Brünger Martin, Buttmann-Schweiger Nina, et al. Guidelines and recommendations for ensuring Good Epidemiological Practice (GEP): a guideline developed by the German Society for Epidemiology. Eur J Epidemiol. 2019;34:301-317.

43 von Elm E, Altman DG, Egger M, Pocock SJ, Gøtzsche PC, Vandenbroucke JP. The Strengthening the Reporting of Observational Studies in Epidemiology (STROBE) statement: guidelines for reporting observational studies. Lancet. 2007;370(9596):1453-7. 